Provided for non-commercial research and education use. Not for reproduction, distribution or commercial use.

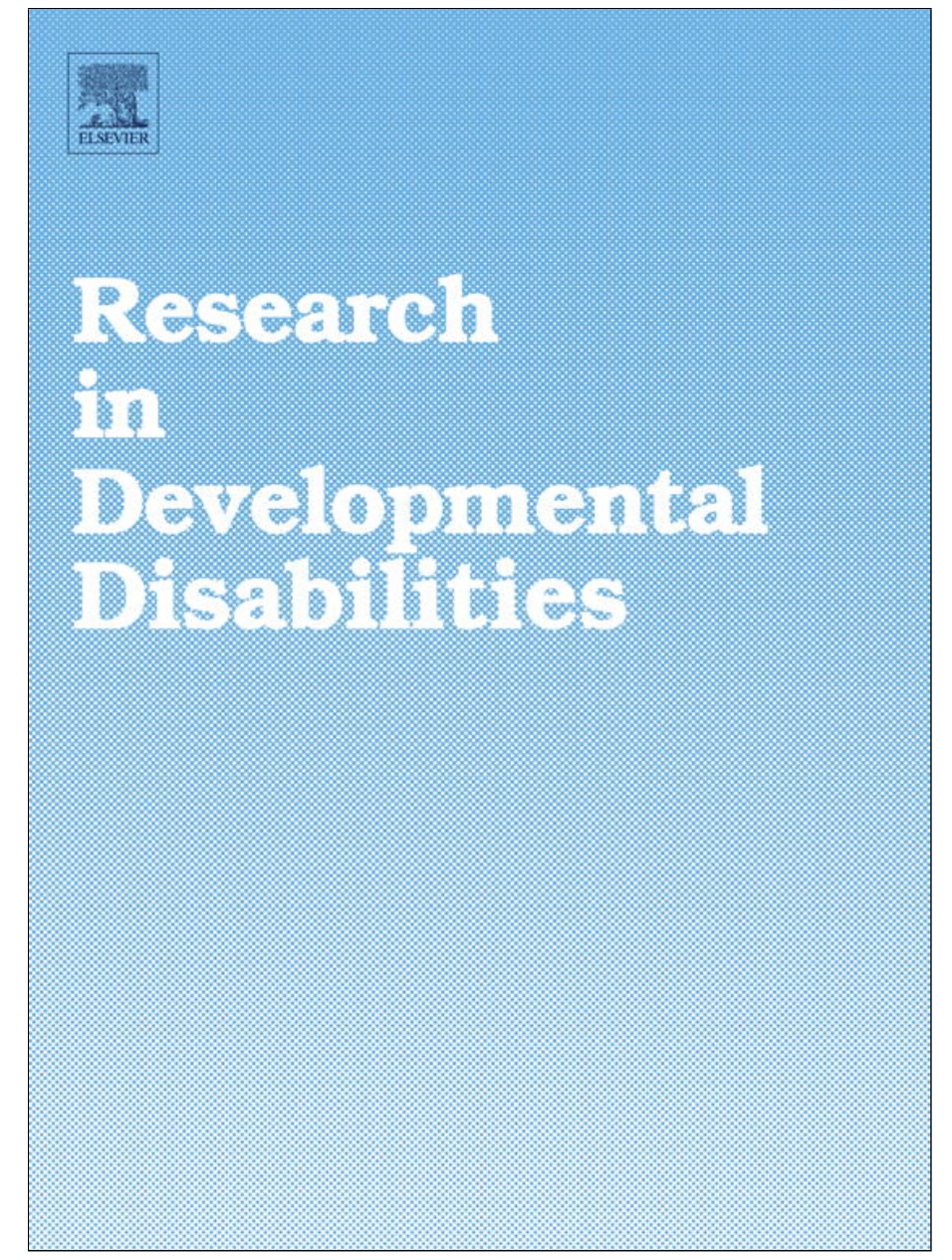

(This is a sample cover image for this issue. The actual cover is not yet available at this time.)

This article appeared in a journal published by Elsevier. The attached copy is furnished to the author for internal non-commercial research and education use, including for instruction at the authors institution and sharing with colleagues.

Other uses, including reproduction and distribution, or selling or licensing copies, or posting to personal, institutional or third party websites are prohibited.

In most cases authors are permitted to post their version of the article (e.g. in Word or Tex form) to their personal website or institutional repository. Authors requiring further information regarding Elsevier's archiving and manuscript policies are encouraged to visit:

http://www.elsevier.com/copyright 


\title{
Patterns of multimorbidity in an older population of persons with an intellectual disability: Results from the intellectual disability supplement to the Irish longitudinal study on aging (IDS-TILDA)
}

\author{
Mary McCarron ${ }^{a}$, Janet Swinburne ${ }^{a}$, Eilish Burke ${ }^{a}$, Eimear McGlinchey ${ }^{a}$, \\ Rachael Carroll ${ }^{\mathrm{a}}$, Philip McCallion ${ }^{\mathrm{b}, *}$ \\ a School of Nursing and Midwifery, Trinity College Dublin, Ireland \\ ${ }^{\mathrm{b}}$ Center for Excellence in Aging and Community Wellness, University at Albany, Albany, NY 12222, USA
}

\section{A R T I C L E I N F O}

\section{Article history:}

Received 30 April 2012

Received in revised form 27 July 2012

Accepted 30 July 2012

Available online

\section{Keywords:}

Intellectual disability

Aging

Health

Multimorbidity

\begin{abstract}
A B S T R A C T
Multimorbidity (2 or more chronic conditions) is being widely studied in older populations and this study explores both the relevance of emerging conceptualizations and the extent to which multimorbidity is a feature of aging in persons with an intellectual disability.

Methods: Data was generated from wave one of the intellectual disability supplement to the Irish longitudinal study on aging (IDS-TILDA) which included 753 persons with an ID aged 40 years and over. Information on the presence of 12 chronic conditions was collected using a standardized protocol administered in face to face interviews with persons with ID and/or their caregivers. Prevalence of multimorbidity was established and patterns were examined using logistic regression models. The patterns of multimorbidity for people with ID that emerged were then compared with those reported for other older adults.

Results: Multimorbidity was established for $71 \%$ of the IDS-TILDA sample with women at highest risk and rates of multimorbidity was high (63\%) even among those aged 4049 years. Eye disease and mental health problems were most often associated with a second condition and the most prevalent multimorbidity pattern was mental health/ neurological disease.

Discussion: Further investigation, attention to mental health issues and the development of treatment guidelines that recognize chronic condition disease load are critical to mitigating the negative impact of multiple chronic conditions and preventing additional disability in adults with ID as they age.
\end{abstract}

(c) 2012 Elsevier Ltd. All rights reserved.

\section{Introduction}

Multimorbidity is defined as the co-occurrence of two or more chronic health conditions in any one person (Barnett et al., 2012). Despite the lack of international assessment standards, multimorbidity is viewed as of increasing concern in the generic aging population (Mercer, Smith, Wyke, O’Dowd, \& Watt, 2009) having major implications for suboptimal quality of life, increased dependency and increased health care costs (Kirchberger et al., 2012). Persons with an intellectual disability (ID) are increasingly living to old age (Kelly \& Kelly, 2011) which means understanding the differences and similarities in understanding the association between chronic disease and increased age has become an important issue in the care of adults with an ID

\footnotetext{
* Corresponding author.

E-mail address: mcclion@albany.edu (P. McCallion).
} 
(Haveman et al., 2010). For the generic population, studies indicate that older people are prone to develop two or more chronic conditions (Kirchberger et al., 2012) with a recent systematic review reporting a range of multimorbidity prevalence rates from $55 \%$ to $98 \%$ in persons aged 65 years and older (Marengoni et al., 2011). It is also increasingly recognized that diseases tend to occur together leading to an emerging interest in the "common pathways" implicated in the clustering of diseases and levels of functional compromise with the potential to help better organize health care responses (Fried, Bandeen-Roche, Kasper, \& Guralnik, 1999; Kirchberger et al., 2012). In the most systematic consideration of multimorbidity in the general older population the diseases found to most commonly occur in combination were hypertension and diabetes and hypertension and stroke. There was also a significant association found between eye disease and diabetes. However, of considerable interest was the encouragement to also consider overlapping mental health and neurological concerns (Kirchberger et al., 2012). This work also resulted in the identification of four multimorbidity patterns: cardiovascular/metabolic, liver/lung/joint/eye, mental/ neurologic and gastrointestinal/cancer disorders with cardiovascular/metabolic, and liver/lung/joint/eye patterns of comorbidity identified as most prevalent (Kirchberger et al., 2012). The usefulness of the Kirchberger multimorbidity patterns in understanding chronic conditions in people with ID remains to be established.

International studies suggest that the health picture for people with ID as they age is even more complex with people with ID reported as having 2.5 times the health problems (Lantman-De, Metsemakers, Haveman, \& Crebolder, 2000). A greater variety of health care concerns is also reported as present as compared to those of the same age and gender in the general population (Haveman et al., 2010; US Department of Health and Human Services, 2002). Central nervous system compromise associated with specific intellectual disabilities (for example, epilepsy and cerebral palsy), may present additional health risks. Small scale studies of specific clinic populations have indicated an increased prevalence of chronic disease with increasing age, such as neurological anomalies; seizure activity; mobility impairments; mental health problems and sensory impairments (for a review see Janicki et al., 2002). A large US study of medical records reported that the frequency of cardiovascular, musculoskeletal and respiratory conditions and sensory impairments increased with age, while neurological, endocrine and mental health problems declined with age and that, despite high levels of risk factors such as obesity, heart related diseases did not appear as prevalent in people with ID as reported in the general population (Janicki et al., 2002). However, there are few large scale studies and none have explored the clustering of major chronic diseases.

The objective of the present study was to describe the extent of multimorbidity in an Irish population of older persons with ID and to explore the patterns in that multimorbidity.

\section{Methods}

Data was drawn from the first wave of data collected as part of the intellectual disability supplement to the Irish longitudinal study on aging (IDS-TILDA). IDS-TILDA is a multi-wave longitudinal study of older adults with ID designed to explore their aging profile, physical and behavioral health, health services needs, psychological health, social networks, living situations and community participation including employment.

Sample: The sample was randomly selected from Ireland's National Intellectual Disability Database (NIDD) which collects information on all people with an ID who are eligible for or receive services with in excess of 26,000 people registered with all levels of ID and in a full range of residential circumstances (Kelly \& Kelly, 2011). Inclusion criteria were: (1) Age $\geq 40$ years with intellectual disability, (2) registered with NIDD, and (3) written consent to participate and/or family/guardian written agreement, where required. Age 40 years was selected to reflect the lower longevity of people with ID to ensure that there would be sufficient subjects for future waves of data collection and because this would provide opportunities to offer insight into aging for those who may age prematurely. The recruited, consented and protocols completed sample was 753 persons with an ID, aged 40 and older; an overall response rate of $46 \%$ comprising persons who represented $8.9 \%$ of the total population aged 40 and over registered on the 2008 NIDD database.

Measures: The first wave data protocol consisted of two main parts with questions largely drawn from existing longitudinal aging studies with a particular emphasis on comparability with The Irish longitudinal study on aging (TILDA) which was collecting data at the same time but whose cohort did not include people with ID (Barrett et al., 2011).

\subsubsection{Pre-interview questionnaire}

A pre-interview questionnaire was sent to each participant before the interview. This questionnaire covers demographical information, health status, healthcare utilization and medication usage. The purpose of this preliminary questionnaire was to give respondents time to source the information required thereby increasing reliability of data.

\subsubsection{Main questionnaire}

The main questionnaire consisted of 17 sections administered as a face-to-face computer assisted interview with each participant. Questions covered additional demographical information, such as type of residence and religion; cognitive health; happiness; physical health; instrumental activities of daily living and support needed to undertake these activities; mental health; employment situation; lifelong learning; day services; behavioral health; social participation; social connectedness; life satisfaction; aging perceptions; personal choices; sources of income; evaluation questions and a final checklist for interviewers. The data reported here is a subsection of the overall data. 
Table 1

Disease categories.

\begin{tabular}{ll}
\hline Disease & Example \\
\hline Hypertension & \\
Eye disease & Age related macular degeneration, glaucoma, cataracts \\
Heart disease & Angina, heart attack, congestive heart failure, open heart surgery \\
Endocrine disease & Thyroid disease, diabetes \\
Joint disease & Arthritis, osteoporosis \\
Lung disease & Asthma, emphysema, chronic COPD \\
Gastrointestinal disease & Stomach ulcers, coeliac disease, chronic constipation \\
Mental health & Anxiety, depression, schizophrenia, psychosis \\
Stroke & Stroke, TIA \\
Cancer & Leukemia, lymphoma \\
Neurological disease & Cerebral palsy, epilepsy, Parkinson's, Alzheimer's \\
Liver & \\
\hline
\end{tabular}

Ethical approval. The IDS-TILDA study received ethics approval from the Faculty of Health Sciences Ethics Committee at Trinity College Dublin and all of the participating services providers $(n=138)$.

\subsection{Data collection}

The presence of chronic health conditions was gathered from participants and/or their caregiver through a pre-interview questionnaire (giving the opportunity to review case files) and confirmed in a standardized computer assisted face to face interview. Each participant/caregiver respondent reported if the individual with ID was ever diagnosed by a doctor/relevant health professional with one or more of 12 chronic health conditions (see Table 1), which are drawn from the Charlson CoMorbidity Index groupings (Charlson, Szatrowski, Peterson, \& Gold, 1994): heart disease; endocrine disease; eye disease; joint disease; stroke; hypertension; cancer; lung disease; gastrointestinal disease; and liver disease.

In addition, given the high prevalence of neurological disease and mental health problems in persons with ID (Haveman et al., 2010; Janicki et al., 2002) and consistent with recommendations by Kirchberger et al. (2012), two other chronic condition groupings were added: neurological diseases (cerebral palsy, epilepsy, multiple sclerosis, Parkinson's diease, spina bifida, muscular dystrophy, Alzheimer's diease, dementia, organic brain syndrome or senility and serious memory impairment) and mental health problems (emotional, nervous or psychiatric condition, hallucinations, anxiety condition, depression, emotional problems, schizophrenia, psychosis, mood swings, and manic depression).

\subsection{Data analysis}

Prevalence, age-standardized (using the WHO standard population 2000-2025) prevalence and the corresponding 95\% confidence interval was calculated for each disease. A logistic regression model for presence of multimorbidity (less than two chronic conditions versus two or more chronic conditions) was estimated adjusted for age (40-49 years, 50-64 years and $65+$ years), gender and level of known ID (mild/moderate and severe/profound) yielding $n=695$.

A two-step process examined the potential to understand multimorbidity patterns. First, because health care in the community for people with ID is likely to be influenced by ideas and practices applicable to the general population, confirmatory factor analysis was used to assess the fit of the Kirchberger et al. (2012) four factor model of multimorbidity patterns. Exploratory factor analysis was also planned to assess whether there was a factor structure more fitting to the patterns among people with ID.

\section{Results}

Of the 753 subjects, $45 \%$ were male and $55 \%$ female. The mean age of the group was 54.75 (sd = 9.56) with 36\% aged $40-$ 49 years; $46 \%$ aged $50-64$ years and $18 \%$ aged 65 years and over. Overall $24 \%$ were classified as having a mild ID, $46 \%$ a moderate ID, 24\% a severe ID, 5\% a profound ID and 5\% were ID unverified. For the purpose of analyses subjects classified with mild and moderate ID were grouped together as were those with severe to profound ID. Those with an unverified level of ID were excluded from the subsequent analyses. The prevalence of the chronic conditions, the age standardized prevalence and the corresponding 95\% confidence interval are presented in Table 2.

Diseases in order of prevalence were: eye disease, mental health disease, neurological disease, gastrointestinal disease, endocrine disease, joint disease, hypertension, heart disease, lung disease, cancer, stroke and liver disease. The highest rate of chronic conditions experienced by an individual was 7 with a median of two conditions and $71.05 \%$ of people displayed multimorbidity (i.e. 2 or more chronic conditions).

In Table 3 the number of conditions is stratified by gender, age and level of ID. Multimorbidity increased from $62.76 \%$ in those aged $40-49$ years to $71.8 \%$ in those aged $50-65$ years and $85.93 \%$ in those aged 65 years and older. In addition, as seen in Table 4, prevalence of eye disease, mental health disease, endocrine disease, joint disease, hypertension, cancer and stroke 
Table 2

Prevalence of twelve chronic conditions.

\begin{tabular}{|c|c|c|c|}
\hline Condition & Crude prevalence & Age standardized prevalence (\%) & 95\% Confidence interval \\
\hline 1. Hypertension & 15.41 & 15.99 & {$[13.03,18.96]$} \\
\hline 2. Eye disease & 51.26 & 51.41 & {$[46.20,56.62]$} \\
\hline 3. Heart disease & 11.82 & 12.11 & {$[9.55,14.66]$} \\
\hline 4. Endocrine & 21.65 & 21.26 & {$[17.95,24.57]$} \\
\hline 5. Joint diseases & 20.45 & 20.92 & {$[17.56,24.28]$} \\
\hline 6. Lung disease & 7.17 & 7.51 & {$[5.48,9.55]$} \\
\hline 7. Gastrointestinal disease & 26.69 & 27 & {$[23.21,30.79]$} \\
\hline 8. Mental health & 47.68 & 48.31 & {$[43.24,53.39]$} \\
\hline 9. Stroke & 2.92 & 3.37 & {$[1.94,4.80]$} \\
\hline 10. Cancer & 4.25 & 4.57 & {$[2.96,6.19]$} \\
\hline 11. Neurological disease & 36.25 & 36.23 & {$[31.87,40.58]$} \\
\hline 12. Liver disease & 0.53 & 0.5 & {$[0.01,0.99]$} \\
\hline
\end{tabular}

Table 3

Number of conditions stratified by gender, age and level of ID.

\begin{tabular}{|c|c|c|c|c|c|c|c|}
\hline \multirow[t]{2}{*}{ \# Conditions } & \multicolumn{2}{|l|}{ Gender } & \multicolumn{3}{|l|}{ Age } & \multicolumn{2}{|l|}{ Level of ID } \\
\hline & Male & Female & 40-49 years & 50-64 years & $65+$ years & Mild/moderate & Severe/profound \\
\hline 0 & $31(9.17 \%)$ & $22(5.3 \%)$ & $28(10.22 \%)$ & $20(5.81 \%)$ & $5(3.7 \%)$ & $38(7.77 \%)$ & $9(4.37 \%)$ \\
\hline 1 & $91(26.92 \%)$ & 74 (17.83\%) & $74(27.01 \%)$ & 77 (22.38\%) & $14(10.37 \%)$ & 106 (21.68\%) & 47 (22.82\%) \\
\hline 2 & 91 (26.92\%) & $105(25.3 \%)$ & $85(31.02 \%)$ & $80(23.26 \%)$ & 31 (22.96\%) & $126(25.77 \%)$ & $51(24.76 \%)$ \\
\hline 3 & $68(20.12 \%)$ & $104(25.06 \%)$ & $54(19.71 \%)$ & $82(23.84 \%)$ & 36 (26.67\%) & $110(22.49 \%)$ & $50(24.27 \%)$ \\
\hline 4 & $28(8.28 \%)$ & $63(15.18 \%)$ & $21(7.66 \%)$ & 46 (13.37\%) & $24(17.78 \%)$ & 57 (11.66\%) & $30(14.56 \%)$ \\
\hline 5 & $18(5.33 \%)$ & $30(7.23 \%)$ & $7(2.55 \%)$ & $26(7.56 \%)$ & 15 (11.11\%) & $31(6.34)$ & $15(7.28 \%)$ \\
\hline 6 & $7(2.07 \%)$ & $13(3.13 \%)$ & $4(1.46 \%)$ & $9(2.62 \%)$ & 7 (5.19\%) & $14(2.86 \%)$ & $4(1.94 \%)$ \\
\hline 7 & $4(1.18 \%)$ & $4(0.96 \%)$ & $1(0.36 \%)$ & $4(1.16 \%)$ & $3(2.22 \%)$ & $7(1.43 \%)$ & $0(0.0 \%)$ \\
\hline Total & 338 & 415 & 274 & 344 & 135 & 489 & 206 \\
\hline
\end{tabular}

were all significantly associated with age. Table 4 also presents odds ratios for reports of each disease by age group, using age 40-49 years as the baseline.

Looking at differences by gender, as can be seen in Table 5, eye disease was significantly higher in women with ID as was joint disease and hypertension. For men and women, mental health concerns, eye disease, neurological disease and gastrointestinal concerns were the highest occurring conditions.

Table 4

Test of independence for each disease and age with corresponding odds ratio.

\begin{tabular}{|c|c|c|c|}
\hline Disease & $\chi_{3}^{2}$ & $p$-Value & OR \\
\hline Eye disease & 14.13 & 0.001 & $\begin{array}{l}1.700^{*} \\
1.921^{*}\end{array}$ \\
\hline Mental health & 11.9 & 0.003 & $\begin{array}{l}1.329 \\
2.072^{*}\end{array}$ \\
\hline Neurological disease & 0.079 & 0.961 & $\begin{array}{l}0.965 \\
0.945\end{array}$ \\
\hline Gastrointestinal disease & 4.35 & 0.114 & $\begin{array}{l}1.259 \\
1.619^{*}\end{array}$ \\
\hline Endocrine & 8.22 & 0.016 & $\begin{array}{l}1.756 \\
1.239\end{array}$ \\
\hline Joint diseases & 12.86 & 0.002 & $\begin{array}{l}1.603^{*} \\
2.463^{*}\end{array}$ \\
\hline Hypertension & 30.03 & $<0.001$ & $\begin{array}{l}2.629^{*} \\
4.795^{*}\end{array}$ \\
\hline Heart disease & 2.18 & 0.347 & $\begin{array}{l}0.945 \\
1.444\end{array}$ \\
\hline Lung disease & 4.78 & 0.092 & $\begin{array}{l}0.983 \\
2.033\end{array}$ \\
\hline Cancer & 12.19 & 0.002 & $\begin{array}{l}2.282 \\
5.733^{*}\end{array}$ \\
\hline Stroke & 21.20 & $<0.001$ & $\begin{array}{r}2.825 \\
14.492^{*}\end{array}$ \\
\hline Liver disease & 1.64 & 0.441 & $\begin{array}{l}0.795 \\
0.000\end{array}$ \\
\hline
\end{tabular}

Note: This table presents the $\mathrm{Chi}^{2}$ test statistic for independence with $3 \mathrm{df}, \chi_{3}^{2}$, and the corresponding $p$-value for each disease and age category and OR indicates the crude odds ratio for the presence of each disease by age where 40-49 years is the baseline group.

* OR is significantly different at the $5 \%$ level of significance from the baseline age group. 
Table 5

Age, gender and level of ID and multimorbidity.

\begin{tabular}{|c|c|c|c|c|c|c|c|c|}
\hline & Log odds & S.E. of $\log$ odds & Wald & df & Sig. & Odds ratio & Lower 95\% CI & Upper 95\% CI \\
\hline Gender (female) & 0.69 & 0.17 & 16.00 & 1 & $<0.001$ & 1.99 & 1.42 & 2.80 \\
\hline Age & & & 17.17 & 2 & $<0.001$ & & & \\
\hline Age (50-64 years) & 0.28 & 0.18 & 2.40 & 1 & 0.122 & 1.33 & 0.93 & 1.90 \\
\hline Age (65+ years) & 1.22 & 0.30 & 17.12 & 1 & $<0.001$ & 3.40 & 1.90 & 6.07 \\
\hline Severe/profound & 0.18 & 0.19 & 0.92 & 1 & 0.339 & 1.20 & 0.83 & 1.74 \\
\hline Constant & 0.19 & 0.17 & 1.32 & 1 & 0.25 & 1.21 & & \\
\hline
\end{tabular}

Note: The baseline categories are male, $40-49$ years and mild/moderate $(n=695)$.

Table 6

Most frequently reported co-occurring pairs of conditions and related odds ratios.

\begin{tabular}{|c|c|c|c|c|c|c|c|}
\hline & Observed & Expected & $\begin{array}{l}\text { Ratio } \\
\mathrm{O} / \mathrm{E}\end{array}$ & $\begin{array}{l}\text { Crude OR } \\
{[99.58 \% \mathrm{CI}]}\end{array}$ & $p$-Value & $\begin{array}{l}\text { Adjusted OR } \\
{[99.58 \% \mathrm{CI}]}\end{array}$ & $p$-Value \\
\hline Eye disease, mental disease & 24.03 & 24.56 & 0.98 & $0.92[0.6,1.42]$ & 0.579 & $0.913[0.567,1.470]$ & 0.584 \\
\hline Eye disease, neurological disease & 19.14 & 19 & 1.01 & $1.02[0.65,1.60]$ & 0.883 & $1.253[0.755,2.082]$ & 0.202 \\
\hline Mental disease, neurological disease & 17.55 & 18.09 & 0.97 & $0.91[0.58,1.43]$ & 0.562 & $0.812[0.502,1.313]$ & 0.215 \\
\hline Gastrointestinal disease, mental disease & 16.26 & 13.22 & 1.23 & $1.86[1.13,3.04]$ & $<0.001$ & $1.729[1.104,2.947]$ & 0.003 \\
\hline $\begin{array}{l}\text { Gastrointestinal disease, neurological } \\
\text { disease }\end{array}$ & 13.24 & 10.23 & 1.29 & $1.88[1.15,3.09]$ & $<0.001$ & $1.444[0.832,2.506]$ & 0.057 \\
\hline Eye disease, joint disease & 12.23 & 10.52 & 1.16 & $1.52[0.88,2.62]$ & 0.027 & $1.579[0.851,2.929]$ & 0.034 \\
\hline Eye disease, gastrointestinal disease & 11.94 & 13.89 & 0.86 & $0.68[0.41,1.10]$ & 0.022 & $0.834[0.471,1.476]$ & 0.362 \\
\hline Endocrine disease, mental disease & 11.37 & 10.57 & 1.08 & $1.2[0.71,2.04]$ & 0.312 & $1.139[0.653,1.989]$ & 0.503 \\
\hline Joint disease, mental disease & 11.08 & 10.02 & 1.11 & $1.3[0.76,2.22]$ & 0.167 & $1.122[0.628,2.004]$ & 0.571 \\
\hline Joint disease, neurological disease & 10.79 & 7.75 & 1.39 & $2.15[1.25,3.70]$ & $<0.001$ & $2.007[1.110,3.628]$ & 0.001 \\
\hline Joint disease, gastrointestinal disease & 8.78 & 5.66 & 1.55 & $2.4[1.37,4.22]$ & $<0.001$ & $2.219[1.187,4.149]$ & $<0.001$ \\
\hline
\end{tabular}

Note: Results of logistic regression models testing the association between pairs of conditions. Odds ratios (OR) were adjusted for age, gender, level of ID and all other diseases.

A logistic regression model (see Table 6 ) using gender, age and level of ID as predictors of reports of multimorbidity was statistically significant, $\chi_{5}^{2}=36.90, p<0.001(n=695)$, explaining between 5.2\% (Cox and Snell $R^{2}$ ) and 7.4\% (Nagelkerke $R^{2}$ ) of the variance in multimorbidity status and correctly classifying $71.2 \%$ of cases. Age and gender made unique statistically significant contributions to the model with the strongest predictor of multimorbidity being age 65+ years. Level of ID did not appear a significant predictor of reports of multimorbidity.

In omnibus tests of model coefficients the finding of $\chi_{5}^{2}=36.9, p<0.001$ indicated that the model that includes age, gender and level ID fit the data significantly better than the null model and the Hosmer and Lemeshow test of differences between observed and predicted values of the response variable agreed; the null model yielded a non-significant value $\left(\chi_{7}^{2}=2.492, p\right.$-value $\left.=0.982\right)$, i.e. the overall model was a better predictor than the null model. Finally a Wald test of the predictive ability of variables in the model supported that gender and age contributed significantly. A female was almost two times more likely to be multimorbid than a male of the same age and level of ID. A person aged between 50 and 64 years was almost 1.5 times more likely to be multimorbid that a person aged between 40 and 49 years and a person aged 65 or over was 3.8 times more likely to be multimorbid than a person aged $40-49$ years.

The presence of Down Syndrome was also examined as a predictor variable. Those with Down Syndrome were less likely to be multimorbid than those who did not have Down Syndrome (OR: 0.683 p-value: 0.051). This association was not significant, however it did approach significance.

Confirmatory factor analysis yielded mixed results regarding the suitability of Kirchberger's multimorbidity patterns for characterizing this dataset. On the one hand an RMSEA of 0.026 with a 0.90 confidence interval $(0.013,0.038)$ and an AGFI of 0.974 are supportive of a good fit but NFI, TLI and CFI of $0.624,0.732$ and 0.805 respectively are not. Nevertheless the sample is portrayed using the four pattern structure in Table 7. As can be seen, the most frequently occurring multimorbidity pattern was mental health/neurologic and cardiovascular/metabolic and liver/lung/joint/eye patterns were not relevant to explaining multimorbidity in people with ID as they age.

Table 7

Persons with ID and the Kirchberger et al. (2012) multimorbidity patterns.

\begin{tabular}{|c|c|c|c|c|c|c|c|}
\hline & Total & Men & Women & $p$-Value & 40-49 years & 50-64 years & $65+$ years \\
\hline Cardiovascular/metabolic & 0 & 0 & 0 & na & 0 & 0 & 0 \\
\hline Liver/lung/joint/eye & 0 & 0 & 0 & na & 0 & 0 & 0 \\
\hline Mental/neurologic & $128(17.0 \%)$ & $59(17.46 \%)$ & $69(16.63 \%)$ & 0.763 & $42(15.33 \%)$ & $58(16.86 \%)$ & $28(20.74 \%)$ \\
\hline Gastrointestinal/cancer & $13(1.73 \%)$ & $7(2.07 \%)$ & $6(1.45 \%)$ & 0.512 & $2(0.73 \%)$ & $6(1.74 \%)$ & $5(3.70 \%)$ \\
\hline
\end{tabular}

Note: Results of $\mathrm{Chi}^{2}$-test for gender and age-group differences. 
Exploratory factor analysis did not yield a better fitting model. First, a Kasier-Meyer-Olkin measure of 0.473 and a borderline correlation matrix raised concerns about the suitability for such analysis and the five factor structure suggested by the analysis consisted of two and three variable factors with poor reliability.

\section{Discussion}

The data here sheds new light on multimorbidity in older adults with ID in Ireland and contributes to a deeper understanding of the complexity of multimorbidity by characterizing the associations between common diseases in this population in multiple ways. Multimorbidity among adults with ID over 40 years was confirmed at a rate of $71.2 \%$, a rate that is much higher than the 58.6\% reported for the generic population over age 65 (Kirchberger et al., 2012). Multimorbidity also appeared to increase with age; those aged 65 or over were 3.4 times more likely to be multimorbid than persons with ID aged 40-49 years. However, it is of concern that multimorbidity was high even in the younger age groups of persons with ID (63\% among those aged 40-49 years) and further investigation to better confirm incidence and prevalence of chronic conditions and the development of treatment guidelines that recognize this chronic condition disease load are critical to mitigating the negative impact of multiple chronic conditions and preventing additional disability in adults with ID as they age.

In the generic population there are conflicting reports on the relationship between multimorbidity and gender. A recent systematic study found that some studies reported no significant gender differences and others that women in older age categories ( 77 years and older and 85 years and older) were more likely to be multimorbid than men (Marengoni, Winblad, Karp, \& Fratiglioni, 2008). In this study a noted gender difference was found with females almost two times more likely to be multimorbid than males, regardless of age. The higher levels of eye, hypertension and particularly joint disease among women with ID and their potential to be associated with other chronic conditions probably explain these differences. The findings here also challenge prior reports for persons with ID that premorbid level of ID confers significant added risk of multimorbidity (see for example, Janicki et al., 2002); in this study, level of ID was not found to be such a significant predictor. The study adds to this literature by highlighting the occurrence of gastrointestinal disease in combination with mental health concerns, joint disease and neurological disease independent of age, gender and level of ID. Such findings encourage greater vigilance for such conditions and less emphasis among physicians on traditional cardiovascular concerns among aging adults with ID; this despite other findings that the population had high levels of obesity (47.1\% were obese or overweight) and seldom engaged in physical activity. The relationship between risk factors and disease incidence and prevalence in people with ID would benefit from longitudinal as opposed to cross-sectional investigation as will consideration of the relatively low levels of smoking and alcohol use reported for this population and the impact of environmental factors such as living locations and self-appraisal of general well-being.

Of most interest are the findings related to mental health concerns and neurological disease and their overlap with eye disease and gastrointestinal and joint diseases. Although level of ID did not appear predictive of multimorbidity, findings on joint disease, neurological and eye disease may be indicative of the continuing role of previously noted central nervous system compromise concerns that are associated with specific intellectual disabilities. In addition, these findings lend support to the growing literature in the generic population on the co-morbid relationship between mental health concerns, neurological disease and other chronic conditions (Kirchberger et al., 2012) and advance recommendations that mental health and neurological conditions should be included in multimorbidity in aging studies (Kirchberger et al., 2012; Maes, Kubera, Obuchowiczwa, Goehler, \& Brzeszcz, 2011).

The patterns of multimorbidity: cardiovascular/metabolic, liver/lung/joint/eye, mental/neurologic and gastrointestinal/ cancer recommended for the design of health care delivery for older adults (Kirchberger et al., 2012) did not appear as much of value for people with ID and highlighted again the relatively low levels of cardiovascular disease. However, the approach did prove valuable in further highlighting the significance of the interplay of mental health and neurological concerns in understanding chronic condition concerns among people with ID as they age.

There are a number of methodological limitations which need to be considered in the interpretation of these results. First, there was not objective testing for diseases; the reliance on self-report may mean that findings of lower frequency of common diseases such as cardiovascular disease, and hypertension may be at least partly due to under recognition and diagnosis. This deserves more rigorous investigation. Second, disease severity was also not assessed and third, results are based on cross-sectional data only, meaning that conclusions may not be drawn on causal relationships among diseases. Finally, the low occurrence in the sample of diseases such as liver disease made both confirmatory and exploratory factor analysis more difficult and needs to be confirmed in other and larger samples before final conclusions are drawn about patterns of multimorbidity. The potential for underdiagnosis of some conditions is again of concern. Nevertheless, analysis of future waves of IDS-TILDA data and of any additional longitudinal studies of the aging of people with ID may help to permit the drawing of such inferences further emphasizing the importance of continued data collection. At the very least these cross-sectional findings offer direction for such future analyses.

\section{Acknowledgements}

We would like to thank the individuals with ID who participated in this study, their families, the services involved, the IDS-TILDA Scientific Advisory Committee and the Intellectual Disability Consultative Groups for their support. We also acknowledge the generous financial support of the Health Research Board and the Department of Health and Children. 


\section{References}

Barnett, K., Mercer, S. W., Norbury, M., Watt, G., Wyke, S., \& Guthrie, B. (2012). Epidemiology of multimorbidity and implications for health care, research, and medical education: A cross-sectional study. The Lancet http://dx.doi.org/10.1016/S0140-6736(12)60240-2.

Barrett, A., Burke, H., Cronin, H., Hickey, A., Kamiya, Y., Kenny, R. A., et al. (2011). Fifty Plus in Ireland 2011: First Results from the Irish Longitudinal Study on Ageing (TILDA). Dublin: Trinity College Dublin.

Charlson, M., Szatrowski, T. P., Peterson, J., \& Gold, J. (1994). Validation of a combined comorbidity index. Journal of Clinical Epidemiology, 47, $1245-1251$.

Fried, L. P., Bandeen-Roche, K., Kasper, J. D., \& Guralnik, J. M. (1999). Association of comorbidity with disability in older women: The women's health and aging study. Journal of Clinical Epidemiology, 52, 27-37.

Haveman, M., Heller, T., Lee, L., Maaskant, M., Shooshtari, S., \& Strydom, A. (2010). Major health risks in aging persons with intellectual disabilities: An overview of recent studies. Journal of Policy and Practice in Intellectual Disabilities, 7, 59-69.

Janicki, M. P., Davidson, P. W., Henderson, C. M., McCallion, P., Taets, J. D., Force, L. T., et al. (2002). Health characteristics and health services utilization in older adults with intellectual disability living in community residences. Journal of Intellectual Disability Research, 46, 287-298.

Kelly, F., \& Kelly, C. (2011). Annual report of the National Intellectual Disability Database Committee. Dublin: Health Research Board.

Kirchberger, I., Meisinger, C., Heier, M., Zimmermann, A. K., Thorand, B., Autenrieth, C. S., et al. (2012). Patterns of multimorbidity in the aged population. Results from the KORA-age study. PLoS One, 7, e30556.

Maes, M., Kubera, M., Obuchowiczwa, E., Goehler, L., \& Brzeszcz, J. (2011). Depression's multiple comorbidities explained by (neuro) inflammatory and oxidative and nitrosative stress pathways. Neuroendocrinol Letters, 32, 7-24.

Marengoni, A., Angleman, S., Melis, R., Mangialasche, F., Karp, A., Garmen, A., et al. (2011). Aging with multimorbidity: A systematic review of the literature. Ageing Research Reviews, 10(4), 430-439.

Marengoni, A., Winblad, B., Karp, A., \& Fratiglioni, L. (2008). Prevalence of chronic diseases and multimorbidity among the elderly population in Sweden. Journal Information, 98.

Mercer, S. W., Smith, S. M., Wyke, S., O’Dowd, T., \& Watt, G. C. M. (2009). Multimorbidity in primary care: Developing the research agenda. Family Practice, 26, 79-80.

US Department of Health and Human Services. (2002). Health centers: America's primary care safety net reflections on success, 2002-2007. USA: US Department of Health and Human Services, Health Resources and Services Administration, Bureau of Primary Health Care.

van Schrojenstein Lantman-De, H. M. J., Metsemakers, J. F. M., Haveman, M. J., \& Crebolder, H. F. J. M. (2000). Health problems in people with intellectual disability in general practice: A comparative study. Family Practice, 17, 405-407. 